\title{
The AGN content of deep radio surveys and radio emission in radio-quiet $A G N$. Why every astronomer should care about deep radio fields
}

\author{
P. Padovani ${ }^{1}$, M. Bonzini ${ }^{1}$, N. Miller ${ }^{2}$, K. I. Kellermann ${ }^{3}$, \\ V. Mainieri ${ }^{1}$, P. Rosati ${ }^{1,4}$, P. Tozzi $^{5}$ and S. Vattakunnel ${ }^{6}$ \\ ${ }^{1}$ European Southern Observatory, \\ Karl-Schwarzschild-Str. 2, D-85748 Garching bei München, Germany \\ email: ppadovan@eso.org \\ ${ }^{2}$ Department of Mathematics and Physical Sciences, Stevenson University, \\ 1525 Greenspring Valley Road, Stevenson, MD 21153-0641, USA \\ ${ }^{3}$ National Radio Astronomy Observatory, \\ 520 Edgemont Road, Charlottesville, VA 22903-2475, USA \\ ${ }^{4}$ Dipartimento di Fisica e Scienze della Terra, Università di Ferrara, \\ Via Saragat 1, I-44122, Ferrara, Italy (current address) \\ ${ }^{5}$ INAF - Osservatorio Astrofisico di Arcetri, Largo E. Fermi, I-50125, Firenze, Italy \\ ${ }^{6}$ INAF, Osservatorio Astronomico di Trieste, Via G. B. Tiepolo 11, I-34131, Trieste, Italy
}

\begin{abstract}
We present our very recent results on the sub-mJy radio source populations at $1.4 \mathrm{GHz}$ based on the Extended Chandra Deep Field South VLA survey, which reaches $\sim 30 \mu \mathrm{Jy}$, with details on their number counts, evolution, and luminosity functions. The sub-mJy radio sky turns out to be a complex mix of star-forming galaxies and radio-quiet AGN evolving at a similar, strong rate and declining radio-loud AGN. While the well-known flattening of the radio number counts below $1 \mathrm{mJy}$ is mostly due to star-forming galaxies, these sources and AGN make up an approximately equal fraction of the sub-mJy sky. Our results shed also light on a fifty-year-old issue, namely radio emission from radio-quiet AGN, and suggest that it is closely related to star formation, at least at $z \sim 1.5-2$. The implications of our findings for future, deeper radio surveys, including those with the Square Kilometre Array, are also discussed. One of the main messages, especially to non-radio astronomers, is that radio surveys are reaching such faint limits that, while previously they were mainly useful for radio quasars and radio galaxies, they are now detecting mostly star-forming galaxies and radio-quiet AGN, i.e., the bulk of the extragalactic sources studied in the infrared, optical, and X-ray bands.
\end{abstract}

Keywords. galaxies: active — galaxies: starburst — radio continuum: galaxies — infrared radiation: galaxies - X-rays: galaxies

\section{Deep radio surveys}

\subsection{The modern radio sky}

The $\mathrm{GHz}$ radio bright ( $\gtrsim 1 \mathrm{mJy}$ ) sky consists mainly of "classical" radio sources, that is radio quasars and radio galaxies. These are active galactic nuclei (AGN) whose radio emission is generated from the gravitational energy associated with a supermassive black hole and emitted through relativistic jets of particles as synchrotron radiation. Below $1 \mathrm{mJy}$ there is an increasing contribution to the radio source population from synchrotron emission resulting from relativistic plasma ejected from supernovae associated with massive star formation in galaxies. Star forming galaxies (SFG), however, appear not to be 
the only component of the faint radio sky, at least down to $\sim 50 \mu \mathrm{Jy}$ at a few $\mathrm{GHz}$ (e.g., Gruppioni et al. 2003, Padovani et al. 2009, 2011, Padovani 2011, Norris et al. 2013), contrary to the (until recently) most accepted paradigm. It turned out that there are still plenty of AGN down there but of the more numerous, radio-fainter type, and therefore not associated to radio quasars and radio galaxies. These AGN, in fact, are of the so-called radio-quiet (RQ) type.

Why should every astronomer care about all this? For a variety of reasons: 1) radio emission, through the so-called "radio-mode" feedback, appears to play a very important role in galaxy evolution (e.g., Croton et al. 2006); 2) RQ AGN reside typically in spiral galaxies, which are still forming stars, and therefore are likely to provide a vital contribution to our understanding of the AGN - galaxy co-evolution issue; 3) radio observations are unaffected by absorption and therefore are sensitive to all types of AGN, independently of their orientation (i.e., type 1s and type 2s); 4) finally, and most importantly, the fact that by going radio faint one starts to detect the bulk of the AGN population (and not only the small minority of radio quasars and radio galaxies) means that radio astronomy is no longer a "niche" activity but is extremely relevant to a whole bunch of extragalactic studies.

\subsection{The issue of radio-quiet $A G N$}

A further point of general interest has to do with RQ AGN. Soon after the discovery of quasars in 1963 it was realized that the majority of them were not as strong radio sources as were the first quasars and were undetected by the radio telescopes of the time: they were "radio-quiet." It was later realized that these sources were actually only "radio-faint." For the same optical power their radio powers were $\approx 3$ orders of magnitude smaller than their radio-loud (RL) counterparts. RQ AGN were until recently normally found in optically selected samples and are characterized by relatively low radio-to-optical flux density ratios and radio powers. It is important to realize that the distinction between the two types of AGN is not simply a matter of semantics: the two classes represent intrinsically different objects, with RL AGN emitting most of their energy over the entire electromagnetic spectrum non-thermally and in association with powerful relativistic jets, while the multi-wavelength emission of RQ AGN is dominated by thermal emission, directly or indirectly related to the accretion disk.

The mechanism responsible for radio emission in RQ AGN has been a matter of debate for the past fifty years. Alternatives have included a scaled down version of the RL AGN mechanism (e.g., Miller et al. 1993, Ulvestad et al. 2005), star formation (Sopp \& Alexander 1991), a black hole rotating more slowly than in RL AGN (Wilson \& Colbert 1995), and many more. This is a non-trivial issue for various reasons: 1$)$ most (>90\%) AGN are RQ; 2) some of the proposed explanations have profound implications on our understanding of AGN physics (jets, accretion, black hole spin, etc.); 3) some others are very relevant for the relationship between AGN and star formation in the Universe (related to "AGN feedback"), which is a hot topic in extragalactic research. We note that it is important to compare the properties of the two AGN classes in the band where they differ most, that is the radio band.

\section{The Chandra Deep Field South}

This is exactly what we did. Our observations were done in the Chandra Deep Field South (CDFS) area, which is part of the Great Observatories Origins Deep Survey (GOODS) and as such one of the most intensively studied regions in the sky. The CDFS is a brainchild of Riccardo Giacconi, who conceived the idea of having the Chandra X-ray 
observatory stare at the same spot of the sky for a long time to reach very faint X-ray fluxes. After the initial $1 \mathrm{Msec}$ (11.6 days; Giacconi et al. 2002), one more was added (Luo et al. 2008). Four Msecs (1.5 months) were finally reached by Xue et al. (2011). More observing time has been granted in the meantime so that a total of 7 Msecs (almost three months) of observing time should be available by the end of 2014 .

Kellermann et al. (2008) used the National Radio Astronomy Observatory (NRAO) Very Large Array (VLA) to obtain $1.4 \mathrm{GHz}$ data, with $8.5 \mu \mathrm{Jy}$ rms noise per 3.5" $\mathrm{x}$ 3.5 " beam, in a field centered on the CDFS, defining a complete sample of 198 radio sources sources reaching $\sim 43 \mu \mathrm{Jy}$ over $0.2 \mathrm{deg}^{2}$. These data were exploited in a series of papers on optical counterparts (Mainieri et al. 2008), X-ray properties (Tozzi et al. 2009), and source populations, evolution, and luminosity functions (LFs) (Padovani et al. 2009, 2011). Miller et al. (2008, 2013) observed the so-called Extended CDFS (E-CDFS), again using the VLA, reaching a somewhat smaller rms over a larger area, namely $\sim 6 \mu \mathrm{Jy} \mathrm{rms}$ noise, and therefore $\sim 30 \mu \mathrm{Jy}$ at $5 \sigma$, in a $2.8 " \mathrm{x} 1.6 "$ beam over $0.3 \mathrm{deg}^{2}$. This resulted in a sample of almost 900 sources. Our group is exploiting these new radio data with the aim of addressing the issues of the faint radio source population and RQ AGN in more detail, given the larger and slightly deeper E-CDFS sample, as compared to the CDFS one. In particular, Bonzini et al. (2012) have identified the optical and infrared (IR) counterparts of the E-CDFS sources, finding reliable matches and redshifts for $\sim 95 \%$ and $\sim 81 \%$ of them respectively, while Vattakunnel et al. (2012) have identified the X-ray counterparts and studied the radio - X-ray correlation for SFG. Finally, Bonzini et al. (2013) have provided reliable source classification.

\subsection{The classification of faint radio sources}

The classification of faint radio sources is complex. First, these objects are quite faint in the optical/near IR regimes. The sources with an optical counterpart have a median magnitude $R \sim 22.8$, but can be hosted in galaxies as faint as $R \sim 27$. Therefore, getting spectra, which can be used for an optical classification is not feasible for the bulk of the objects. But even if we had optical spectra for all our sources we would still have problems since, as also remarked by a few speakers at this conference (e.g., Laura Trouille) a single band only gives a biased view of the properties of AGN and the optical band is the worst one, being strongly affected by obscuration. Indeed, there are quite a few examples of optically boring sources where the AGN is detected only in the X-ray band (i.e., some of the so-called X-ray Bright Optically Normal Galaxies; XBONGs). One then needs to use all the multi-wavelength information available for the E-CDFS, which is substantial, to figure out what a radio source really is, looking for AGN in the IR and X-ray bands as well.

Bonzini et al. (2013) have used a modified version of the classification scheme used by Padovani et al. (2011) to disentangle SFG, RQ, and RL AGN. In short, they identify RL AGN using the so-called $q_{24}$ parameter, which is defined by $\log f_{24 \mu m} / f_{1.4 G H z}$, while RQ AGN are distinguished from SFG according to their IRAC (near-IR) colours and X-ray power. Finally, other parameters were examined to check the classification and sort out possible outliers. These included: the presence of an inverted radio spectrum $\left(\alpha_{\mathrm{r}}<0\right.$, where $\left.S_{\nu} \propto \nu^{-\alpha}\right)$, possible Very Long Baseline Array (VLBA) detections, broad/high excitation lines in the optical spectra, Polycyclic Aromatic Hydrocarbon (PAH) features, X-ray absorption and variability.

\subsection{The faint radio source population}

Thanks to this careful classification, we now have what we believe is the most accurate determination of the source population of faint radio sources. Fig. 1 shows the preliminary 


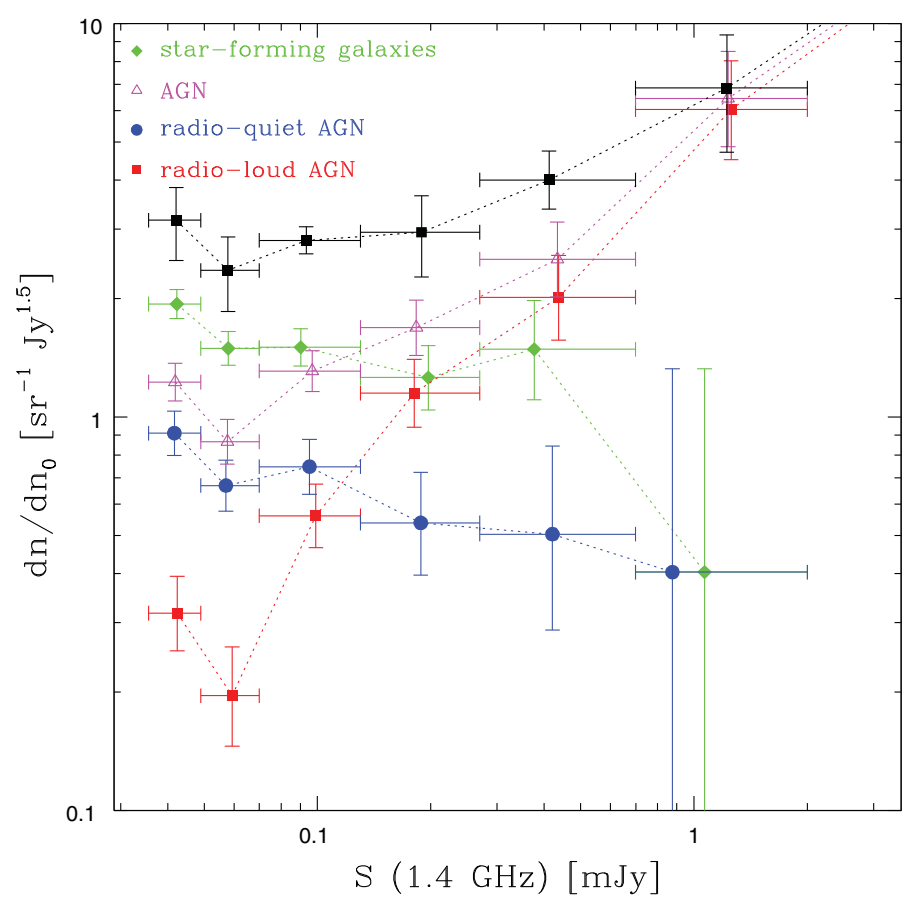

Figure 1. Preliminary Euclidean normalized E-CDFS source counts: whole sample (black squares), SFG (green diamonds), all AGN (magenta triangles), radio-quiet AGN (blue circles), and radio-loud AGN (red squares). Error bars correspond to $1 \sigma$ Poisson errors (Gehrels 1986).

Euclidean normalized number counts for our sample, along with the population subsets (Padovani et al., in preparation). Fig. 2, adapted from Bonzini et al. (2013), plots the relative fractions of the various classes as a function of radio flux density. One can see that AGN dominate at large flux densities ( $\gtrsim 1 \mathrm{mJy}$ ) but SFG become the main population below $\approx 0.1$ mJy. Similarly, RL AGN are the predominant type of AGN above $0.1 \mathrm{mJy}$ but their contribution drops fast at lower flux densities. In more detail, AGN make up $43 \pm 4 \%$ of sub-mJy sources, going from $100 \%$ of the total at $\sim 10 \mathrm{mJy}$, down to $39 \%$ at the survey limit. SFG, on the other hand, which represent $57 \pm 3 \%$ of the sub-mJy sky, are missing at high flux densities but become the dominant population below $\approx 0.1$ mJy, reaching $61 \%$ at the survey limit. RQ AGN represent $26 \pm 6 \%$ (or $60 \%$ of all AGN) of sub-mJy sources but their fraction appears to increase at lower flux densities, where they make up $73 \%$ of all AGN and $\approx 30 \%$ of all sources at the survey limit, up from $\approx 6 \%$ at $\approx 1$ mJy. These results are in good agreement with those of Padovani et al. (2011). The strong message is that below $0.1 \mathrm{mJy}$ the radio sky, which at large flux densities is characterized by the prevalence of synchrotron radiation associated with powerful jets, is instead dominated by star-formation-related processes.

\subsection{Evolution and luminosity functions}

Having redshifts for the majority of our sources, we can also study the LFs and evolution of faint radio sources. The most general approach to do this is to perform a maximum likelihood fit of an evolving LF to the observed distribution in luminosity and redshift (see details in Padovani et al. 2011). This method makes maximal use of the data and is free from arbitrary binning, although it is model dependent. 


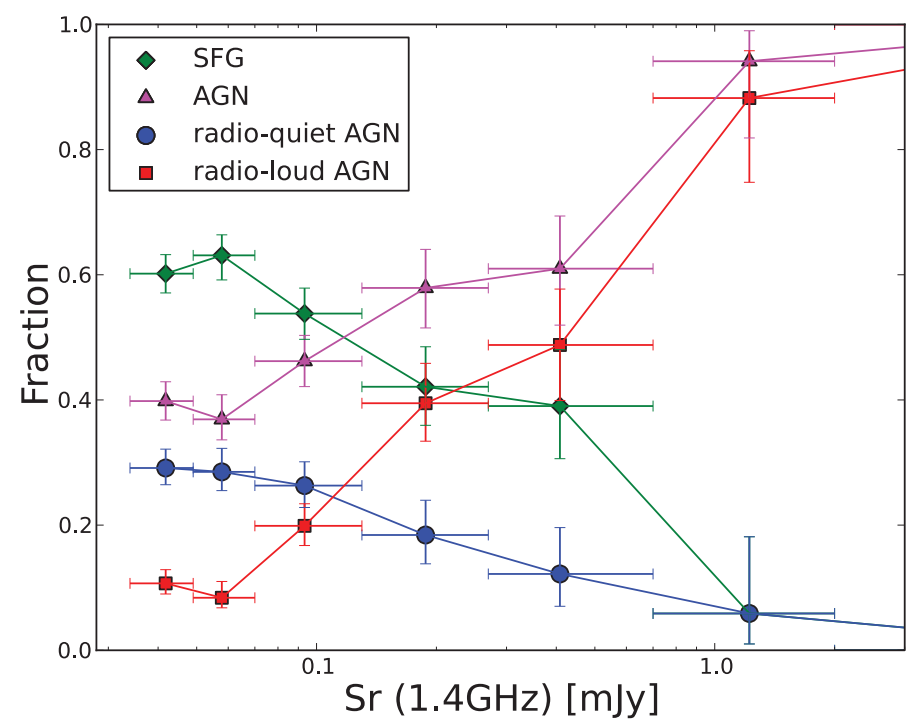

Figure 2. Relative fraction of the various classes of E-CDFS sources as a function of radio flux density: SFG (green diamonds), all AGN (magenta triangles), radio-quiet AGN (blue circles) and radio-loud AGN (red squares). Error bars correspond to $1 \sigma$ Poisson errors (Gehrels 1986). Adapted from Bonzini et al. (2013).

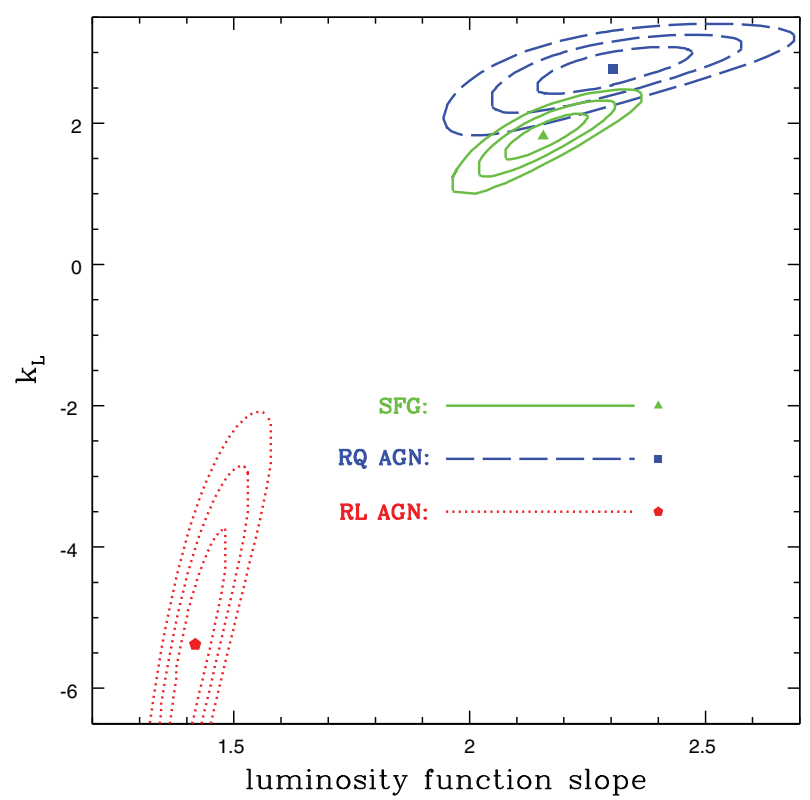

Figure 3. Preliminary maximum likelihood confidence contours $(1 \sigma, 2 \sigma$, and $3 \sigma)$ for the E-CDFS sample assuming a pure luminosity evolution of the type $\propto(1+z)^{k_{L}}$ and a single power-law for the LF. The best fit values for the various classes are indicated by the different symbols.

Fig. 3 plots preliminary maximum likelihood confidence contours $(1 \sigma, 2 \sigma$, and $3 \sigma)$ under the simple assumptions of a pure luminosity evolution of the type $\propto(1+z)^{k_{L}}$ and a single power-law for the LF. $k_{L}>0$ indicates positive evolution, i.e., the higher the redshift the larger the power, while $k_{L}<0$ indicates negative evolution, i.e., the 
higher the redshift the smaller the power. RQ AGN and SFG occupy the same region of parameter space (strong positive evolution and steep LF), while RL and RQ AGN are totally disconnected, the former displaying negative evolution and a flat LF. Note that, even considering a more complicated picture, as suggested by a more detailed analysis of the data (i.e., a dual power-law LF: Padovani et al. in prep.), RQ AGN and SFG still evolve similarly in the radio band. In particular, the derivation of the local LF confirms that RL AGN have a much flatter LF than RQ ones and shows that the RQ AGN LF seems to be an extension of the SFG LF at higher radio powers (see also Padovani et al. 2009).

\section{Radio emission in radio-quiet AGN}

Our results suggest very close ties between star formation and radio emission in RQ AGN at $z \sim 1.5-2$, the mean redshift of our sources, since their evolution is similar to that of SFG and their LF appears to be an extension of the SFG LF. Furthermore, radio emission in the two classes of AGN appears to have a different origin. If RQ AGN were simply "mini RL" AGN, in fact, they would have to share the evolutionary properties of the latter and their LF should also be on the extrapolation of the RL LF at low powers. Neither of these is borne out by our data. This conclusion agrees with our previous results (Padovani et al. 2011) and Kimball et al. (2011). This result is further confirmed by the comparison of the star formation rates (SFRs) derived from the far-IR and radio luminosities, assuming that all the radio emission is due to SF. For RQ AGN and SFG the two SFR estimates are fully consistent, while for RL AGN the agreement is poor due to the large contribution of the relativistic jet to their radio luminosity (Bonzini et al., this volume).

\section{A revolution in radio astronomy}

The Square Kilometre Array (SKA; http://www.skatelescope.org) will offer an observing opportunity extending well into the nanoJy regime with unprecedented versatility (we stress that at present we have reached $\sim 15 \mu \mathrm{Jy}$ at $1.4 \mathrm{GHz}$ ). First science with the so-called $\mathrm{SKA}_{1}$ is scheduled early in the next decade. The LOw Frequency ARray (LOFAR) has already started operations and will carry out large area surveys at 15, 30, 60, 120 and $200 \mathrm{MHz}$ (Morganti et al. 2009), opening up a whole new region of parameter space at low radio frequencies. The VLA has been upgraded and the new instrument, the Jansky Very Large Array (JVLA; https://science.nrao.edu/facilities/vla), has hugely improved capabilities and MERLIN, which is now e-MERLIN (http://www.emerlin.ac.uk/), has greatly improved sensitivity. Many other radio telescopes are currently under construction in the lead-up to the SKA including the Australian Square Kilometre Array Pathfinder (ASKAP; http://www.atnf.csiro.au/projects/askap/), Apertif (http://www.astron.nl/general/apertif/apertif), and MeerKAT (http://www.ska.ac.za/meerkat). These projects will survey the sky faster than has been possible with existing radio telescopes producing surveys covering large areas of the sky down to fainter flux densities than presently available (see Norris et al. 2013 for details).

As an example, simple modelling based on our results shows that a radio survey reaching $\sim 1 \mu \mathrm{Jy}$ at $1.4 \mathrm{GHz}$ will need to cover only $\sim 30 \mathrm{deg}^{2}$ to detect a number of "potential" AGN larger than those currently known, while $\sim 200 \mathrm{deg}^{2}$ should reveal about one million such sources. The Evolutionary Map of the Universe (EMU), one of the ASKAP projects, expects to reach flux densities similar to those of the E-CDFS over $\sim 3 / 4$ of the sky, detecting more than 70 million sources, about half of which will be "potential" AGN (Norris et al. 2011). The "potential" here is important because, as detailed above, the classification of faint radio sources requires a great deal of ancillary, multi-wavelength 
information, which will not be easy to get at very faint levels (Padovani 2011) or over very large areas.

\section{Conclusions}

We have shown that the GHz sub-mJy sky is a complex mix of evolving star-forming galaxies and radio-quiet AGN and negatively evolving radio-loud AGN. Contrary to what used to be the predominant view, AGN still make up $\sim 40 \%$ of the faint radio sky, although the deep $(<0.1 \mathrm{mJy})$ radio sky appears to be dominated by star forming galaxies and therefore stellar processes. Radio-quiet AGN are also very important, accounting for $\sim 60 \%$ of all sub-mJy AGN and $\sim 25 \%$ of the total radio faint population. Radio surveys, which were in the past only useful to study radio-loud AGN, have reached so deep that they are now dominated by the same galaxies detected by IR, optical and X-ray surveys. As a result, the next generation radio surveys, which will produce samples of tens of millions of sources, will be an increasingly important component of multiwavelength studies of galaxy evolution. Finally, by studying the evolution and luminosity functions of faint radio sources, we have found a close relationship between star formation and radio emission in radio-quiet $\mathrm{AGN}$ at $z \sim 1.5-2$, which is confirmed by the very similar star formation rates obtained independently in the radio and far-IR bands.

\section{References}

Bonzini, M., Mainieri, V., Padovani, P., et al. 2012, ApJS, 203, 15

Bonzini, M., Padovani, P., Mainieri, V., et al. 2013, MNRAS, 436, 3759

Croton, D. J., Springel, V., White, S. D. M., et al. 2006, MNRAS, 365, 11

Gehrels, N. 1986, ApJ, 303, 336

Giacconi, R., Zirm, A., Wang, J., et al. 2002, ApJS, 139, 369

Gruppioni, C., Pozzi, F., Zamorani, G., et al. 2003, MNRAS, 341, L1

Kellermann, K. I., Fomalont, E. B., Mainieri, V., Padovani, P., Rosati, P., Shaver, P., Tozzi, P., \& Miller, N. 2008, ApJS, 179, 71

Kimball, A. E., Kellermann, K. I., Condon, J. J., Ivezić, Ž., \& Perley, R. A. 2011, ApJ (Letters) 739, L29

Luo, B., Bauer, F. E., Brandt, W. N., et al. 2008, ApJS, 179, 19

Mainieri, V., Kellermann, K. I., Fomalont, E. B., et al. 2008, ApJS, 179, 95

Miller, P., Rawlings, S., \& Saunders, R. 1993, MNRAS, 263, 425

Miller, N. A., Fomalont, E. B., Kellermann, K. I., et al. 2008, ApJS, 179, 114

Miller, N. A., Bonzini, M., Fomalont, E. B., et al. 2013, ApJS, 205, 13

Morganti, R., Rottgering, H., Snellen, I., et al. 2009, in: G. Heald \& P. Serra (eds.), Panoramic Radio Astronomy: Wide-field 1-2 GHz research on galaxy evolution, Gröningen, The Netherlands, published online at http://pos.sissa.it/cgi-bin/reader/conf.cgi? confid $=89,40$

Norris, R. P., Hopkins, A. M., Afonso, J., et al. 2011, PASA, 28, 215

Norris, R. P., Afonso, J., Bacon, D., et al. 2013, PASA, 30, 20

Padovani, P., Mainieri, V., Tozzi, P., Kellermann, K. I., Fomalont, E. B., Miller, N., Rosati, P., \& Shaver, P. 2009, ApJ, 694, 235

Padovani, P. 2011, MNRAS, 411, 1547

Padovani, P., Miller, N., Kellermann, K. I., Mainieri, V., Rosati, P., \& Tozzi, P. 2011, ApJ, 740, 20

Sopp, H. M. \& Alexander, P. 1991, MNRAS, 251, 14P

Tozzi, P., Mainieri, V., Rosati, P., et al. 2009, ApJ, 698, 740

Ulvestad, J. S., Antonucci, R. R. J., \& Barvainis, R. 2005, ApJ, 621, 123

Vattakunnel, S., Tozzi, P., Matteucci, F., et al., 2012, MNRAS, 420, 2190

Wilson, A. S. \& Colbert, E. J. M. 1995, ApJ, 438, 62

Xue, Y. Q., Luo, B., Brandt, W. N., et al. 2011, ApJS, 195, 10 